\title{
Associação entre Renda, Características Clínicas e Angiográficas de Pacientes Submetidos a Intervenção Coronária Percutânea
}

\author{
Carlos Augusto Homem de Magalhães Campos', Alexandre Schaan de Quadros², Dulce I. Welter², \\ Fernanda Camozzatto ${ }^{2}$, Denise Rovinski², Rogério Sarmento-Leite ${ }^{2}$, Carlos Gottschall ${ }^{2}$
}

\section{RESUMO}

Introdução: Há grande interesse científico com relação ao status socioeconômico e à saúde populacional, e vários estudos demonstraram influência dessas variáveis na evolução clínica dos pacientes. A presente análise avalia a influência da renda do indivíduo na distribuição dos fatores de risco e nas características clínicas e angiográficas em pacientes submetidos a intervenção coronária percutânea. Método: Os pacientes submetidos a intervenção coronária percutânea em um centro terciário, entre dezembro de 2007 e março de 2008, foram incluídos prospectivamente. A informação relativa à renda foi coletada diretamente com o paciente por um dos pesquisadores do estudo. $\mathrm{Na}$ presente análise, os indivíduos foram divididos em quartis, de acordo com seus respectivos rendimentos mensais. Resultados: Foram incluídos 400 pacientes com média de idade de $61,01 \pm 10,36$ anos, sendo $64,7 \%$ do sexo masculino e $24,6 \%$, diabéticos. A maioria apresentava angina estável $(54,9 \%)$, e não houve diferenças entre os grupos quanto às comorbidades. A renda associou-se ao porcentual de pacientes do sexo masculino $(48 \%$ no primeiro quartil vs. $81,3 \%$ no último quartil; $\mathrm{P}<0,001)$ e às relações ponderoestaturais $(P<0,05)$, mas não houve associação com o índice de massa corporal médio. Observamos também associação significativa entre renda e utilização de stents farmacológicos, sendo as seguintes taxas do primeiro ao quarto quartis: $0,1 \%, 1,9 \%$ e $7,7 \%(P=0,003)$. Conclusão: Neste estudo, observamos associação entre renda e gênero, características antropométricas e utilização de stents farmacológicos. Não houve associação entre renda e fatores de risco, história médica pregressa e características angiográficas dos pacientes.

DESCRITORES: Renda. Angioplastia. Stents. Iniquidade social.

\begin{abstract}
Association Between Income, Clinical and Angiographic Characteristics in Patients Undergoing Percutaneous Coronary Intervention

Background: There is great scientific interest with respect to socioeconomic status and population health, and several studies have shown the influence of these variables on clinical outcome of patients. The present study assesses the influence of income on the distribution of risk factors, clinical and angiographic characteristics in patients undergoing percutaneous coronary intervention. Method: Patients undergoing percutaneous coronary intervention in a tertiary center from December 2007 to March 2008 were prospectively included. The information on income was directly collected from the patient by one of the investigators. In this analysis, subjects were divided into quartiles according to their respective monthly income. Results: Four hundred patients with mean age of $61.01 \pm 10.36$ years, $64.7 \%$ males and $24.6 \%$ diabetics were included. A majority had stable angina (54.9\%), and there were no differences between groups regarding comorbidities. Income was associated with the percentage of male patients $(48 \%$ in the first quartile vs. $81.3 \%$ in the last quartile; $P<0.001)$, the weight and height $(P<0.05)$, but there was no association with mean body mass index. We also observed a significant association between income and use of drug-eluting stents with the following rates from first to fourth quartile: $0,1 \%, 1.9 \%$ and $7.7 \%(P=0.003)$. Conclusion: In this study, there was an association between income and gender, anthropometric characteristics and use of drug-eluting stents. There was no association between income and risk factors, medical history and angiographic characteristics of patients.
\end{abstract}

KEY-WORDS: Income. Angioplasty. Stents. Social inequity.

\footnotetext{
1 Serviço de Hemodinâmica e Cardiologia Intervencionista - Hospital Ibiapaba/CEBAMS - Barbacena, MG, Brasil.

${ }^{2}$ Instituto de Cardiologia do Rio Grande do Sul - Fundação Universitária de Cardiologia/IC-FUC - Porto Alegre, RS, Brasil.

Correspondência: Alexandre Schaan de Quadros. Unidade de Pesquisa do IC-FUC - Av. Princesa Isabel, 370 - Santana - Porto Alegre, RS, Brasil - CEP 90620-001

E-mail: alesq@terra.com.br

Recebido em: 16/12/2009 • Aceito em: 13/2/2010
} 
A tualmente há grande interesse científico com relação ao status socioeconômico e à saúde populacional. Alguns autores demonstraram que, em um mesmo sistema de saúde, a menor renda está relacionada a aumento de três a sete vezes ${ }^{1}$ da mortalidade. Da mesma forma, a morbidade também é mais acentuada entre os pobres, com séries demonstrando índices de internações hospitalares até quatro vezes maiores. ${ }^{2}$ Além disso, aqueles pacientes de melhor situação econômica tendem a fumar menos e a ser menos obesos, e, no que tange à cardiologia intervencionista, têm mais acesso a procedimentos invasivos, são atendidos com infarto agudo com menor tempo de isquemia e possuem maior acesso a tecnologias mais modernas em saúde. ${ }^{3-5}$

No Brasil essas questões são particularmente importantes, uma vez que o País apresenta uma das piores distribuições de renda do mundo. ${ }^{6}$ Bassanesi et al. ${ }^{7}$ já demonstraram que a mortalidade precoce por doença cardiovascular pode ser até 3,3 vezes maior entre extremos de renda, sendo pior nos menos favorecidos. Como essas diferenças podem interferir na interpretação dos desfechos em pesquisa clínica, a presente análise procura comparar, de acordo com a renda do indivíduo, os fatores de risco, as características angiográficas e o tratamento adotado entre os pacientes submetidos a intervenção coronária percutânea.

\section{MÉTODO}

Os pacientes considerados para inclusão no estudo foram submetidos a intervenção coronária percutânea no Instituto de Cardiologia/Fundação Universitária de Cardiologia do Rio Grande do Sul (IC-FUC) entre dezembro de 2007 e março de 2008. Trata-se de uma instituição de ensino médico, referência para tratamento cardiovascular de alta complexidade com ampla abrangência socioeconômica. Nesta comparação, não houve qualquer restrição quanto a quadro clínico de admissão, idade, anatomia coronária ou número de stents implantados. Os critérios de exclusão foram procedimentos sem sucesso, eventos cardiovasculares maiores na internação, participação em ensaios clínicos randomizados e recusa em assinar o termo de consentimento livre e esclarecido.

\section{Coleta de dados}

Todos os dados foram coletados por um dos pesquisadores durante a internação índice, seguindo o preenchimento de formulários previamente padronizados. A coleta incluiu características clínicas, resultados de exames laboratoriais, dados do procedimento invasivo e renda mensal dos participantes. A informação relacionada à renda de cada indivíduo foi obtida diretamente do paciente por um dos pesquisadores, após esclarecimento da finalidade de utilização exclusiva dessa informação para pesquisa e compromisso de total confidencialidade por parte dos pesquisadores.

\section{Definições}

Índice de massa corporal (IMC) foi definido pela fórmula: peso em quilogramas dividido pela altura ao quadrado (em metros). Os pacientes foram considerados obesos quando seu IMC foi igual ou maior a 30 .

As indicações para intervenção coronária percutânea foram divididas em três síndromes principais: angina estável, considerada quando havia dor desencadeada por esforço, isquemia silenciosa documentada por prova funcional ou com equivalente anginoso; diagnóstico de infarto agudo do miocárdio com supradesnível do segmento ST se houvesse elevação persistente de ST maior que $1 \mathrm{~mm}$ em duas derivações contíguas ou bloqueio de ramo esquerdo novo ao eletrocardiograma; e, por último, síndromes coronárias agudas sem supradesnível do segmento ST. Neste grupo, a angina instável foi definida como dor em repouso, de início recente ou progressiva, sem os critérios eletrocardiográficos citados anteriormente; quando associada a elevação de marcadores de necrose miocárdica, foi considerada infarto do miocárdio sem supradesnível do segmento ST.

\section{Análise de dados}

Os indivíduos foram divididos em quartis, de acordo com seus respectivos rendimentos mensais. Esses grupos foram comparados com relação a fatores de risco, comorbidades, e características clínicas, angiográficas e relacionadas ao procedimento.

As variáveis contínuas foram descritas como média \pm desvio padrão e foram comparadas por ANOVA. As variáveis categóricas foram representadas como sua porcentagem e comparadas com teste de qui-quadrado de associação linear. As análises foram realizadas com programa estatístico SPSS 13.0, e P $<0,05$ foi considerado estatisticamente significante para todos os testes.

\section{RESULTADOS}

Entre dezembro de 2007 e março de 2008, 400 pacientes foram submetidos a intervenção coronária percutânea na instituição. A mediana da renda encontrada foi de $\mathrm{R} \$ 800,00$. Esses indivíduos foram divididos em quartis, de acordo com a renda: remuneração até $R \$ 450,00(n=100)$; entre $R \$ 450,00$ e $R \$ 800,00$ ( $n=105)$; entre $R \$ 800,00$ e $R \$ 1.500,00$ ( $n=103)$; e superior a $R \$ 1.500,00(n=92)$.

\section{Comparação das características clínicas}

No global, a média de idade foi de 61,01 \pm 10,36 anos, sendo $64,7 \%$ do sexo masculino, $77,7 \%$ hipertensos e 24,6\% diabéticos, e a apresentação clínica foi, em sua maioria (54,9\%), a angina estável. Conforme demonstrado na Tabela 1, a idade tendeu a ser mais avançada no primeiro $(63,18 \pm 10,32)$ e no terceiro quartis $(61,27 \pm 10,31)$ que no segundo $(59,9 \pm 10,27)$ 


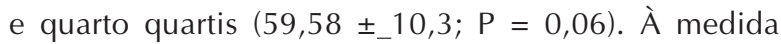
que houve incremento da renda, maior foi a participação do sexo masculino entre os pacientes tratados, variando de $48 \%$ a $81,3 \%$ a porcentagem de homens $(\mathrm{P}<0,001)$. Não houve diferenças entre os grupos para os outros antecedentes clínicos ou comorbidades dos pacientes, tais como hipertensão, diabetes, insuficiência renal, insuficiência cardíaca, tabagismo, revascularização miocárdica prévia, infarto do miocárdio prévio, antecedentes familiares ou dislipidemia (Tabela 1). A presença de depressão foi quase duas vezes mais frequente naqueles com renda inferior a $R \$ 800,00$, mas sem alcançar significância estatística.

\section{Relações ponderoestaturais entre os grupos}

O aumento da remuneração mensal foi relacionado a progressivo aumento do peso dos pacientes, variando de 71,68 $\pm 13,54 \mathrm{~kg}$, nos indivíduos com rendimentos inferiores a $R \$ 450,00$, a 79,22 $\pm 13,34 \mathrm{~kg}$, naqueles com renda superior a R\$ $1.500,00$, conforme demonstrado na Tabela 2. Da mesma forma, houve incremento da altura dos pacientes à medida que o salário aumentou: $1,63 \pm 0,08 \mathrm{~m}$ no primeiro quartil, 1,65 $\pm 0,08 \mathrm{~m}$ no segundo quartil, 1,67 $\pm 0,09 \mathrm{~m}$ no terceiro quartil, e 1,69 $\pm 0,07$ no quarto quartil $(\mathrm{P}<0,001)$. Assim, como houve incremento progressivo do peso e da altura dos grupos, quando comparamos os IMCs não encontramos diferença significativa entre os grupos. No entanto, quando pesquisamos a presença de obesidade nesses indivíduos, houve tendência a divergência entre os quartis pela baixa incidência de obesidade no primeiro quartil (15\%), sendo de $29,5 \%$ no segundo quartil, de $25,2 \%$ no terceiro quartil, e de $24,2 \%$ no quarto quartil $(P=0,09)$. $\mathrm{Na}$ média, em todos os percentis, a população deste estudo mostrou-se em estágio de sobrepeso (IMC entre 25-30), e o IMC médio global foi de 27,46 $\pm 4,55$.

\section{Características dos tratamentos percutâneos}

Observa-se, na Tabela 3, que a média de utilização de stents por paciente bem como seu diâmetro e comprimento foram semelhantes entre os quatro grupos de pacientes. Também não houve diferença significativa entre os vasos abordados na intervenção percutânea. No entanto, a utilização de stents farmacológicos cresceu significativamente com o aumento da renda: $0 \%, 1 \%, 1,9 \%$ e $7,7 \%$ do primeiro ao quarto quartil, respectivamente $(P=0,003)$.

TABELA 1

Características clínicas de acordo com a renda

\begin{tabular}{|c|c|c|c|c|c|}
\hline & $\begin{array}{c}\text { Renda } \\
\text { inferior a } \\
\text { R\$ 450,00 } \\
(n=100)\end{array}$ & $\begin{array}{c}\begin{array}{c}\text { Renda } \\
\text { entre }\end{array} \\
\text { R\$ 450,00 e } \\
\text { R\$ 800,00 } \\
(n=105)\end{array}$ & $\begin{array}{c}\begin{array}{c}\text { Renda } \\
\text { entre }\end{array} \\
\text { R\$ 800,00 e } \\
\text { R\$ } 1.500,00 \\
(n=103)\end{array}$ & $\begin{array}{c}\text { Renda } \\
\text { superior a } \\
\text { R\$ 1.500,00 } \\
(\mathbf{n}=92)\end{array}$ & $\mathbf{P}$ \\
\hline Idade, anos \pm DP & $63,18 \pm 10,32$ & $59,9 \pm 10,27$ & $61,27 \pm 10,31$ & $59,58 \pm 10,3$ & 0,06 \\
\hline Sexo masculino, $\%$ & 48 & 61 & 69,9 & 81,3 & $<0,001$ \\
\hline Hipertensão, \% & 77 & 84,8 & 76,7 & 71,4 & 0,15 \\
\hline Diabetes, \% & 30 & 25,7 & 21,4 & 20,9 & 0,4 \\
\hline ICP prévia, \% & 23 & 26,7 & 32 & 33 & 0,36 \\
\hline CRM prévia, \% & 15 & 17,1 & 12,6 & 17,6 & 0,75 \\
\hline IAM prévio, \% & 43 & 41,9 & 44,7 & 33 & 0,36 \\
\hline Dislipidemia, \% & 56 & 56,2 & 54,4 & 56 & 0,99 \\
\hline \multicolumn{6}{|l|}{ História familiar de } \\
\hline DAC, $\%$ & 46 & 55,2 & 50,5 & 58,2 & 0,33 \\
\hline $\mathrm{IC}, \%$ & 2 & 1,9 & 4,9 & 2,2 & 0,51 \\
\hline Tabagismo, \% & 29 & 34,3 & 27,2 & 22 & 0,29 \\
\hline Insuficiência renal, \% & 2 & 1,9 & 1,9 & 1,1 & 0,96 \\
\hline Depressão, \% & 13 & 15,2 & 7,8 & 7,7 & 0,21 \\
\hline DPOC, \% & 5 & 1 & 3,9 & 3,3 & 0,41 \\
\hline Quadro clínico, \% & & & & & 0,1 \\
\hline Angina estável & 54 & 44,8 & 60,2 & 61,5 & \\
\hline Angina instável/IAMSST & 30 & 33,3 & 25,2 & 17,6 & \\
\hline IAMST & 16 & 21,9 & 17,6 & 20,9 & \\
\hline
\end{tabular}

CRM = cirurgia de revascularização miocárdica; DAC = doença arterial coronária; DP = desvio padrão; DPOC = doença pulmonar obstrutiva crônica; IAM = infarto agudo do miocárdio; IAMSST = infarto agudo do miocárdio sem supradesnível do segmento ST; IAMST = infarto agudo do miocárdio com supradesnível do segmento ST; IC = insuficiência cardíaca; ICP = intervenção coronária percutânea. 
TABELA 2

Relações ponderoestaturais de acordo com a renda

\begin{tabular}{|c|c|c|c|c|c|}
\hline & $\begin{array}{c}\text { Renda } \\
\text { inferior a } \\
\text { R\$ 450,00 } \\
(n=100)\end{array}$ & $\begin{array}{c}\begin{array}{c}\text { Renda } \\
\text { entre }\end{array} \\
\text { R\$ 450,00 e } \\
\text { R\$ 800,00 } \\
(n=105)\end{array}$ & $\begin{array}{c}\begin{array}{c}\text { Renda } \\
\text { entre }\end{array} \\
R \$ \mathbf{8 0 0 , 0 0} \text { e } \\
R \$ 1.500,00 \\
(n=103)\end{array}$ & $\begin{array}{c}\text { Renda } \\
\text { superior a } \\
\mathbf{R} \$ 1.500,00 \\
(\mathbf{n}=92)\end{array}$ & $\mathbf{P}$ \\
\hline Peso, kg & $71,68 \pm 13,54$ & $76,61 \pm 15,1$ & $77,58 \pm 15,32$ & $79,22 \pm 13,34$ & 0,003 \\
\hline Altura, m & $1,63 \pm 0,08$ & $1,65 \pm 0,08$ & $1,67 \pm 0,09$ & $1,69 \pm 0,07$ & $<0,001$ \\
\hline IMC & $26,68 \pm 4,24$ & $27,96 \pm 5,08$ & $27,69 \pm 4,5$ & $27,57 \pm 4,21$ & 0,22 \\
\hline Obesidade, \% & 15 & 29,5 & 25,2 & 24,2 & 0,09 \\
\hline
\end{tabular}

$\mathrm{IMC}=$ índice de massa corporal.

TABELA 3

Características angiográficas e do procedimento conforme a renda

\begin{tabular}{|c|c|c|c|c|c|}
\hline & $\begin{array}{c}\text { Renda } \\
\text { inferior a } \\
\text { R\$ 450,00 } \\
(n=100)\end{array}$ & $\begin{array}{c}\text { Renda } \\
\text { entre } \\
\text { R\$ 450,00 e } \\
\text { R\$ 800,00 } \\
(n=105)\end{array}$ & $\begin{array}{c}\text { Renda } \\
\text { entre }\end{array}$ & $\begin{array}{c}\text { Renda } \\
\text { superior a } \\
\text { R\$ } 1.500,00 \\
(\mathbf{n}=92)\end{array}$ & $\mathbf{P}$ \\
\hline Stent/paciente & $1,18 \pm 0,38$ & $1,17 \pm 0,4$ & $1,17 \pm 0,39$ & $1,12 \pm 0,32$ & 0,71 \\
\hline Comprimento do stent & $16,34 \pm 5,7$ & $17,34 \pm 6,09$ & $16,71 \pm 5,14$ & $17,27 \pm 5,55$ & 0,62 \\
\hline Diâmetro do stent & $3,18 \pm 0,69$ & $3,11 \pm 0,32$ & $3,17 \pm 0,37$ & $3,06 \pm 0,41$ & 0,54 \\
\hline Vaso tratado & & & & & 0,17 \\
\hline DA & 48 & 45,7 & 28,2 & 42,9 & \\
\hline CX & 18 & 18,1 & 25,2 & 15,4 & \\
\hline CD & 32 & 32,4 & 44,7 & 39,5 & \\
\hline Múltiplos vasos & 2 & 3,8 & 1,9 & 2,2 & \\
\hline Stent utilizado, \% & & & & & 0,003 \\
\hline SF & 0 & 1 & 1,9 & 7,7 & \\
\hline SC & 100 & 99 & 98,1 & 91,3 & \\
\hline
\end{tabular}

\section{DISCUSSÃO}

Neste centro terciário de atenção à cardiologia de alta complexidade, com grande abrangência socioeconômica, a análise dos pacientes submetidos a intervenções coronárias percutâneas com relação à renda demonstrou, como principais achados, que: (1) não houve associação entre os rendimentos mensais e a presença de comorbidades; (2) houve maior participação porcentual do sexo feminino quanto menor $\mathrm{O}$ salário; (3) houve demonstração de maior estatura e peso com incremento financeiro, sem incremento no IMC médio; e (4) apesar da semelhança angiográfica os stents farmacológicos foram mais utilizados entre os mais abastados.
A renda individual pode influenciar hábitos e, dessa forma, poderia modificar o status de saúde. Na presente população, não houve qualquer associação entre menores rendimentos e maior adoecimento, medido pela presença de comorbidades. O estudo Boston Area Community Health $(\mathrm{BACH})$ demonstrou forte correlação entre a presença de diabetes e o perfil socioeconômico dos pacientes. ${ }^{8}$ De forma semelhante, estudo conduzido por Mackenbach et al., ${ }^{9}$ em 22 países da Europa, demonstrou que pior nível socioeconômico foi diretamente relacionado a maior uso de tabaco e obesidade. No entanto, os achados referentes a aspectos socioeconômicos, hábitos e desfechos estão sujeitos a regionalismos. Corroborando essa 
afirmação, esse mesmo estudo de Mackenbach et al. ${ }^{9}$ demonstrou grandes variações nos países europeus com relação a saúde, hábitos e características sociais. Meta-análise de Kondo et al., ${ }^{10}$ com inclusão de 59.509.857 indivíduos de estudos de coorte e de 1.280.211 indivíduos de estudos seccionais cruzados, sugere que o adoecimento pode estar relacionado a desequilíbrios sociais, porém apresenta grandes variações regionais. Essas afirmações reforçam a importância desta análise, que registra uma população brasileira tratada em um contexto de mundo real, com perfis financeiros diversos.

Fato curioso foi a tendência à diferença na idade para a realização de angioplastia nas diferentes faixas de renda. Com relação ao sexo, essas diferenças foram ainda mais acentuadas, em que as mulheres corresponderam a mais da metade dos procedimentos no primeiro quartil e a menos de $20 \%$ no quarto quartil, com aumento progressivo entre o primeiro e último quartis. A causa para tamanha diferença não nos parece clara, mas já foi encontrada previamente. Grande estudo populacional canadense, que incluiu 51.591 pacientes com diagnóstico de infarto agudo do miocárdio, demonstrou que à medida que se aumentava a renda, havia maior participação proporcional de pacientes do sexo masculino. Da mesma forma, nessa população, houve oscilação de idade entre os grupos, com significância estatística. ${ }^{5}$

Chama a atenção que, com o aumento da renda, os indivíduos tiveram também progressivo aumento em sua estatura e peso médios, sem afetar a diferença de seu IMC. De fato, os ganhos financeiros parecem estar associados a maior desenvolvimento ponderoestatural. Recente estudo de Fernald et al. ${ }^{11}$ randomizou 1.793 indivíduos de uma população mexicana de baixa renda para ajuda financeira imediata ou tardia. Após 10 anos de seguimento houve aumento de $1,5 \mathrm{~cm}$ com o apoio financeiro imediato acessado pelo índice peso-por-altura $(P=0,029) .{ }^{11}$ Além disso, em nossa população, de forma intuitiva, houve também tendência a diferença na presença de obesidade (IMC $\geq 30$ ) pela menor incidência no primeiro quartil, que compreende os indivíduos com renda até $\mathrm{R}$ \$ 450,00.

Há correlação direta entre diâmetros dos stents utilizados e diâmetro de referência do vaso. O comprimento do stent e o comprimento da lesão também guardam a mesma proporção. Dessa forma, observamos semelhança angiográfica entres os grupos analisados. Também não houve diferença na média de utilização de stents por paciente. E apesar dessas semelhanças e também dos antecedentes clínicos, como diabetes ou apresentação clínica, observamos significativa diferença na utilização de stents farmacológicos à medida que há melhora socioeconômica, variando de 0 a $7,7 \%(P=0,003)$. Esse dado pode ser explicado pelo fato de, atualmente, no Brasil, a utilização de stents eluidores de medicação estar sujeita à forma de abrangência do plano de saúde privado, como também pelo fato de o sistema público nacional não abranger essa tecnologia. Essa realidade não está restrita a nosso País, afetando também nações mais abastadas e com melhor distribuição de renda. Hannan et al. ${ }^{12}$ demonstraram que pacientes de menor renda têm menor chance de ser tratados com stents farmacológicos nos Estados Unidos (OR 0,80; IC 95\% 0,68-0,93). Outro estudo norte-americano demonstrou que os stents farmacológicos foram menos utilizados em pacientes sem seguro saúde (OR 0,44; IC 95\% 0,37-0,53) e com seguro governamental (OR 0,73; IC 95\% 0,67-0,80) que naqueles com seguro saúde privado. ${ }^{13}$

Uma das principais limitações da comparação do presente estudo com outros relatos da literatura reside no fato de termos utilizado somente a renda dos pacientes para estimar seus perfis socioeconômicos. Outros estudos populacionais adicionam a esse fator dados como moradia, educação, densidade populacional, envelhecimento, fecundidade e mortalidade infantil. Porém é sabido que a renda está diretamente ligada a classe social, acesso à educação, moradia, etc. Por esse motivo, acreditamos ter realizado adequada estratificação social na população tratada em nosso centro.

Como mensagem final, devemos ter em mente que na busca por caminhos para a produção de conhecimentos não devemos ficar restritos aos limites tradicionais dos estudos: procedimentos metodológicos não são mais que posturas historicamente construídas para se atingir objetivos. Lembremos que, como citou Michel Foucault, "a medicina moderna é uma prática social que possui uma tecnologia do corpo" e a atenção a esse fato deve guiar nossa prática.

\section{CONCLUSÃO}

Nesta população brasileira atendida em um centro terciário de atenção cardiovascular a renda não modificou o grau de adoecimento dos indivíduos. O ganho financeiro esteve associado a maior número de intervenções no sexo masculino, maiores valores antropométricos e com utilização mais frequente de stents farmacológicos.

\section{CONFLITO DE INTERESSES}

Os autores declararam inexistência de conflito de interesses relacionado a este manuscrito.

\section{REFERÊNCIAS}

1. Pappas G, Queen S, Hadden W, Fisher G. The increasing disparity in mortality between socioeconomic groups in the United States, 1960 and 1986. N Engl J Med. 1993;329: 103-9.

2. Billings J, Zeitel L, Lukomnik J, Carey TS, Blank AE, Newman L. Impact of socioeconomic status on hospital use in New York City. Health Aff (Millwood). 1993;12:162-73.

3. Sheifer SE, Rathore SS, Gersh BJ, Weinfurt KP, Oetgen WJ, 
Breall JA, et al. Time to presentation with acute myocardial infarction in the elderly: associations with race, sex, and socioeconomic characteristics. Circulation. 2000;102:1651-6.

4. Philbin EF, McCullough PA, DiSalvo TG, Dec GW, Jenkins $\mathrm{PL}$, Weaver WD. Socioeconomic status is an important determinant of the use of invasive procedures after acute myocardial infarction in New York State. Circulation. 2000;102 Suppl 3:III-107-III-15.

5. Alter DA, Naylor C, David A, Peter Tu, Jack V. Effects of socioeconomic status on access to invasive cardiac procedures and on mortality after acute myocardial infarction. $N$ Engl J Med. 1999;341:1359-67.

6. Polanczyk C, Ribeiro JP. Coronary artery disease in Brazil: contemporary management and future perspectives. Heart. 2009;95:870-6.

7. Bassanesi SL, Azambuja MI, Achutti A. Mortalidade precoce por doenças cardiovasculares e desigualdades sociais em Porto Alegre: da evidência à ação. Arq Bras Cardiol. 2008; 90:403-12.

8. Link CL, McKinlay JB. Disparities in the prevalence of diabetes: is it race/ethnicity or socioeconomic status? Results from the
Boston Area Community Health (BACH) survey. Ethn Dis. 2009;19:288-92.

9. Mackenbach JP, Stirbu I, Roskam A-JR, Schaap MM, Menvielle G, Leinsalu M, et al., for the European Union Working Group on Socioeconomic Inequalities in Health. Socioeconomic inequalities in health in 22 European countries. N Engl J Med. 2008;358:2468-81.

10. Kondo N, Sembajwe G, Kawachi I, van Dam RM, Subramanian SV, Yamagata Z. Income inequality, mortality, and self rated health: meta-analysis of multilevel studies. BMJ. 2009;339: b4471.

11. Fernald LCH, Gertler PJ, Neufeld LM. 10-year effect of Oportunidades, Mexico's conditional cash transfer programme, on child growth, cognition, language, and behaviour: a longitudinal follow-up study. Lancet. 2009;374:1997-2005.

12. Hannan EL, Racz M, Walford G, Clark LT, Holmes DR, King SB $3^{\text {rd }}$, et al. Differences in utilization of drug-eluting stents by race and payer. Am J Cardiol. 2007;100:1192-8.

13. Kao J, Vicuna R, House JA, Rumsfeld JS, Ting HH, Spertus JA. Disparity in drug-eluting stent utilization by insurance type. Am Heart J. 2008;156:1133-40. 